This item was submitted to Loughborough's Research Repository by the author.

Items in Figshare are protected by copyright, with all rights reserved, unless otherwise indicated.

Towards symbiotic human-robot collaboration: human movement intention recognition with an EEG

PLEASE CITE THE PUBLISHED VERSION

https://doi.org/10.31256/ukras19.14

PUBLISHER

UK-RAS Network

VERSION

VoR (Version of Record)

LICENCE

CC BY-NC-ND 4.0

REPOSITORY RECORD

Buerkle, Achim, Niels Lohse, and Pedro Ferreira. 2019. "Towards Symbiotic Human-robot Collaboration: Human Movement Intention Recognition with an EEG”. Loughborough University.

https://hdl.handle.net/2134/14135588.v1. 


\title{
Towards Symbiotic Human-Robot Collaboration: Human Movement Intention Recognition with an EEG
}

\author{
Achim Buerkle, Niels Lohse, Pedro Ferreira \\ Intelligent Automation Centre, The Wolfson School of Mechanical, Electrical and Manufacturing Engineering, Loughborough University
}

\begin{abstract}
In order to meet the trend of customers demanding more customised and complex products, human workers and robots need to collaborate in closer proximity. Working in shared environments raises safety concerns of humans getting injured by robots. Current safety systems are mostly vision based and detect movement after it has started. This work proposes the use of an electroencephalography (EEG) which measures the brainwaves in order to detect a worker's intention to move. This is expected to provide 0.5 seconds gain for the system to react in advance of the actual movement. In this paper the details on how EEG sensors can be integrated to detect intentions and how these can be extrapolated using machine learning techniques, are presented. The ultimate vision is to deliver an early warning system to enhance existing safety systems.
\end{abstract}

Keywords: Human-Robot Collaboration, Symbiotic Assembly Systems, Robot Safety, EEG, Movement-Intention Recognition, Machine Learning

\section{INTRODUCTION}

The consumer market for manufactured goods is currently following a trend from mass produced goods towards individual and highly customised products. Simultaneously due to shorter lifecycles of products and a higher product variety, manufacturing goods becomes more complex [1].

Conventional robots are designed for low mix production repeatedly coping with a high payload at a high speed while providing consistent quality. This, however, conflicts with the aforementioned trend towards manufacturing highly customised goods [1] [2].

Human workers strengths, on the other hand, are the cognitive skills, adaptability to changes and the ability of making difficult decisions with incomplete data [3].

An opportunity to cope with the demands of high adaptability and high complexity in manufacturing can be the symbiosis of robots and human workers in a confined workspace. A symbiosis typically combines the benefits of two systems. This, however, requires clear and transparent communication channels and awareness of all members in the system [3].

While most research focuses on robots replicating human activities and movements, in a human-robot collaboration environment both parties are expected to perform tasks simultaneously which are related to their characteristic strengths. Fully automated systems, on one hand, can achieve a high production volume at a low-level product complexity. Manual systems, on the other hand, typically produce complex products at a low volume. Therefore, human-robot collaboration could change manufacturing towards producing high complexity goods at a high production volume [3].

Consequently, human workers and robots need to work in close proximity to each other. These "fenceless", shared manufacturing environments raise safety concerns, as robots lack abilities to detect humans, whilst being able to operate at high speed and at high levels of torque. In order to ensure human worker's safety, there are video-based systems available which will stop/slow down a robot once a human worker is approaching the robot and at risk of getting injured [4]. Shutting down a robot as a safety feature, however, should be seen as 'ultima ratio' - the final argument/solution. A better approach is to react beforehand by communicating the intention meaning the willingness of performing a task before the actual execution [5].

There are concepts for Robot-Human communication such as using Augmented Reality glasses to show robot movement animations to human workers in advance, in order to highlight the robot's intentions [1].

However, communicating human intentions to a robot is rather difficult. Therefore, a concept to apply electroencephalography (EEG)-based (Brainwave-measuring) systems to detect human intentions to perform motor-movements is investigated. Instead of replicating the movement intention with a robotic arm, the measured intention will be used as means of communication. In order to validate, that EEG-based sensors can be integrated in a system, it is essential to accurately detect human movement intentions. The idea is to establish a new mechanism to enhance current video-based safety systems, by taking advantage of detecting intentions before the actual execution.

\section{BACKGROUND}

Robots seem to be an essential part of today's manufacturing industry with 2.6 million robots in operation worldwide in 2019 and increase of $13 \%$ every year [2].

However, the level of human-robot interaction in such systems is still fairly low, despite recent advances in robot technology [6]. The interactions between humans and robots range from working in parallel, in sequence or jointly. Thus, one can argue that the interactions are dependent on the level of expected proximity between the two. A very low-level interaction is the human-robot coexistence. Both share the same working 
environment while performing different tasks which do not interfere. In such situations, safety systems are required to avoid collisions, however human-robot communication is restricted to "START" / "STOP"-commands only [7]. In a human-robot assistance scenario the activities of both humans and robots need to be synchronised. Also, a communication needs to be established to enable interoperability. The relationship of humans and robots can be viewed as Client and Server interaction. Robots are supposed to serve workers without cognitive capabilities, hence they're human-guided. [4] The highest level of interaction is the human-robot collaboration by jointly executing tasks. This is also due to the fact that it's the most intense and challenging way of interaction. Since both workers and robots do not only share the same task but also interact within the same process, interoperability is required on a detailed level [3].

Apart from both workers and robots needing to be aware of each other, timing of each task is also essential. This leads to the need of situational, goal-oriented planning as well as being aware of each member's current and future tasks or intentions. Possible interferences of tasks need to be anticipated and solved in order to establish a safe collaboration. Therefore, robots are also required to have cognitive skills [7].

In order to establish safety during a collaborative operation, ISO 10218-1 distinguishes between four levels of collaboration and associated means of risk reduction [8]. The first one is a safety-rated monitored stop, meaning that there will be no robot motion once a human operator is entering in a collaborative work space. A second operation is hand-guiding which will only allow robot motion through direct input of the operator. The third clause contains the definition of speed and separation monitoring. Therefore, robot motion is enabled when the separation distance is above minimum separation distance. Finally, there is power and force limiting by design or control. In that case robots are designed to only impart limited static or dynamic forces in the event of a human-robot contact [9]. However, these safety features are the 'ultima ratio' - the final solution to prevent hazards. Organising tasks and communicating intentions from operators to robots and robots to operators beforehand could avoid creating high riskscenarios which would trigger the safety systems to interfere.

According to Gustavsson et al. (2018) there are three main approaches to robot-human communication. One is augmented reality (AR) which overlays digital information onto cameracaptured real-world objects. The technology provides information where it's needed. A second approach is Text-ToSpeech (TTS) which allows robots to provide understandable audible messages for human workers. While this technology is mostly used in devices such as satellite-navigation, there are also opportunities to use it in a manufacturing environment. A third approach is Pick-by-light. A small lamp installed on each storage container indicates which part or tool a human worker should pick. Although this system is not very flexible, it has been in use in modern warehouses [6].

Human-to-robot communication, on the other hand, often focuses on natural or intuitive human communication channels. While haptic controls such as a joystick to guide a robot were used in assistance or coexistence scenarios, they are less applicable in human-robot collaboration. Automatic speech recognition (ASR), on the other hand, allows humans to communicate in a natural way with the robot, similar to humanhuman interactions. In contrast to haptic controls, humans communicate without removing hands or their focus from their current task. Gesture recognition is not only restricted to hands but can also mean nodding the head in order to indicate an affirmative decision. Both vision-based, and glove-based technologies allow the system to locate a human worker's position as well as receiving commands such as highlighting an object by pointing at it. Overall, best results were achieved when combining different channels [10].

Safety systems tend to rely on vision-based systems in humanrobot collaborative manufacturing [4]. Their main purpose is to help avoiding collisions or limiting impact forces to a level that they won't cause injuries. The reason why vision-based safety systems are so popular is because of their affordable price, high flexibility and easy tailoring[4].

While electroencephalography (EEG) has been used to analyse brain behaviour for a few decades, due to recent developments regarding mobility, it can now also used in an industrial environment [5]. Mohammed and Wang (2018) established training sessions for human operators to think of commands. The measured brain activity-patterns were translated into robot commands. Similar to vision based communication, this allows an operator to perform an individual task, while at the same time controlling the robot in a human-robot assistance scenario [11].

\section{PROBLEM DESCRIPTION}

As described in Section II, there is a trend towards humanrobot collaboration in industrial assembly processes. Humanrobot collaboration is more than human-robot coexistence and human-robot assistance. Human-robot collaboration requires both to share the same working environment while performing tasks which are assigned according to the required, characteristic strength of either humans or robots [4].

The main advantages of human-robot collaboration will be the ability of coping with high production volume while assembling increasingly more complex products [3].

However, these shared working environments raise safety concerns due to the close proximity. Therefore, human workers and robots need to be in a constant feedback loop which ensures awareness of each member in the system in order to avoid collisions or any negative interference. The main purpose of these safety system is to keep human workers safe and to prevent possible injuries. Current safety systems solutions are mostly vision-based. They detect the current location of a human worker as well as the performed movement. Typically, movements are detected after their execution has started, which is only possible after certain timespan which is required to process the images.

The question that arises is if it is possible to predict movements before they occur. This would provide the safety system with faster reaction times and allow for higher leeway on robot operations. Based on the processed images, there are 
approaches to predict future motions by using statistical methods and estimated probabilities. The accuracy of these predictions, however, decreases with the complexity of the assembly task [4].

An opportunity to physically measure human arm movement intentions and therefore enhancing/extending existing visionbased safety system can be the usage of EEG-measured brain waves. Liu and Wang (2017) state that the human brain is always analysing and evaluating motions before executing them [12].

Therefore, it is possible to detect intentions such as human arm movement by recognising patterns in the EEG, as highlighted in Figure 1 [5].
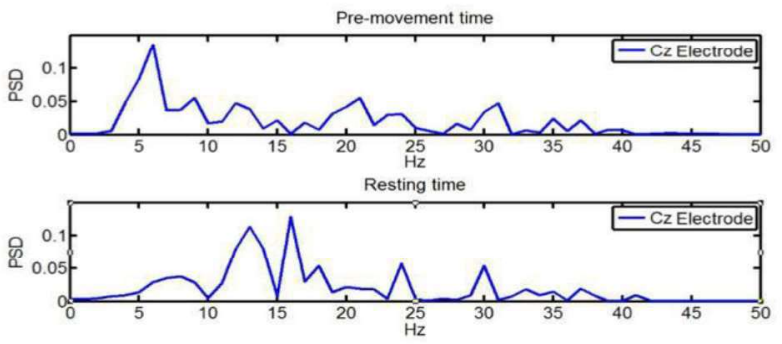

Figure 1 Pre-movement patterns [13]

Generally, there are two neuro-physiological phenomena that can be detected before a voluntary action occurs. The first one, sending weak signals, is called Bereitschaftspotenzial (readiness potential) and occurs up to 1.5 seconds before the actual movement. The second one, sending a stronger, more easily measurable signal through EEG, can be detected up to 0.5 seconds before the movement [13]. In order to gain a timerelated advantage, the time to process the EEG data, including feature-extraction, classification, evaluation by contextualising and finally, giving the feedback command, needs investigation whether it is faster than video-based systems. However, the data-stream transferred by EEGs is presumably smaller than the data-stream which is processed in video-based safety systems. This could also offer the opportunity of achieving a faster processing performance.

Another challenge that should be considered apart from processing and reaction time is accuracy. Safety systems typically require a high Recall (retrieving all intentions) and then a high accuracy. The main purpose of this bias is to avoid False Negatives, meaning that the safety system should rather cause a false alarm, than missing an actual hazardous situation. Therefore, the safety systems should consider this bias when modelled to ensure a similar behaviour to existing systems. There is a clear potential to assess EEG as a potential solution for predicting motion, which can be used in safety system, but would also have wider applications on human-robot collaborations, as it would provide an interfacing path that currently does not exist. Nevertheless, it is also important to state that EEG-based experiments are mostly performed in quiet and sterile laboratories which are intended to protect an experiment from external influences as good as possible. In an industrial environment, on the other hand, there are various sources of external stimuli such as a high noise level, machines performing movements and other sensor-disturbing influences. Typically, an EEG is measuring micro-voltage levels whereas a human worker could stand next to a machine running at thousands of volts. These influences also need further investigation and considered before an EEG-based system can be applied in an industrial environment.

\section{METHODOLOGY FOR DETECTING HUMAN INTENTIONS TO MOVE}

In order to extend existing safety systems, it is essential to measure worker's movement intentions. An EEG allows to detect patterns; however, these need to be mapped with actions to differentiate between being idle, having the intention to move and finally the movement itself. Therefore, the main approach is first, to detect movement intentions before then optimising classification accuracies. Finally, the time-related advantage over video-based systems needs to be validated. The general process for an integrated movement intention recognition with an EEG would be (1) measuring brainwaves at certain locations before (2) pre-processing the signal by using filters to reduce the noise. Bousetta et al. (2017) examined channels (Figure 3 ) to detect movement intentions. The channels AF3, AF4, F3 and F4 were used[13].

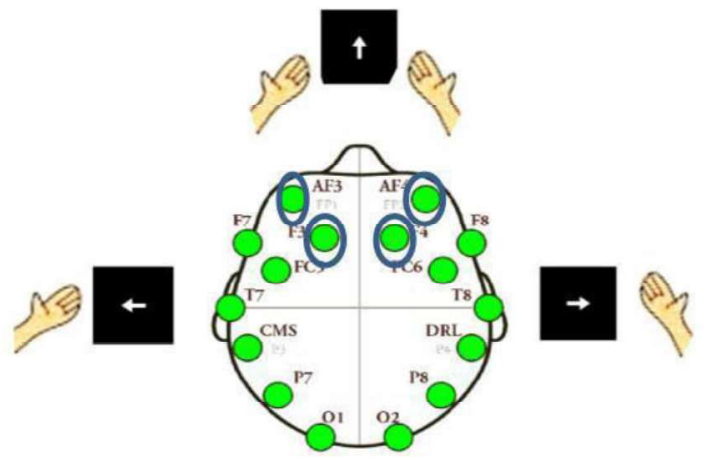

Figure 2 Responsible EEG-channels [5]

In a third step, a feature extraction needs to be performed to reduce the dimensionality of the features. Typically, sensorimotor rhythms that can be measured before and during an arm movement, are frequency bands of $8 \mathrm{~Hz}-12 \mathrm{~Hz}$ and $12 \mathrm{~Hz}-22 \mathrm{~Hz}$ which can be filtered with a Butterworth-filter. After applying a Fast Fourier Transformation, distinct signals can be detected [5], [13].

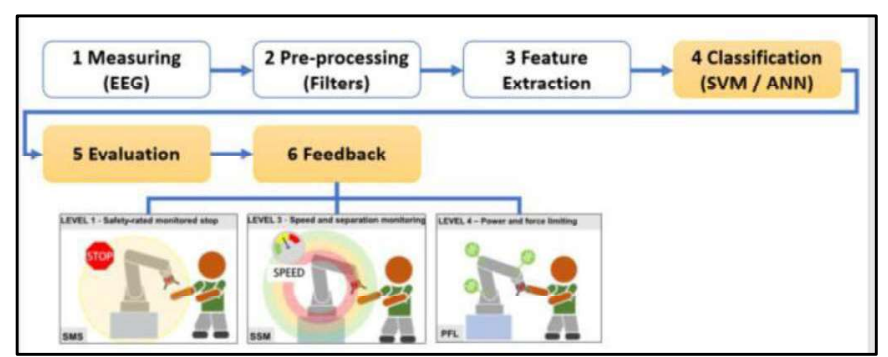

Figure 3 Integrated EEG-measurement in Process

Finally, a classification is performed (4). For each arm there are the aforementioned classes: Idle, intention and movement. 
Bousetta et al. (2017) used a Support-Vector Machine (SVM) as a state of the art-classifier [5]. Planelles et. Al. (2014) also obtained highest accuracies with SVMs compared to other statistical methods such as k-nearest neighbours [13]. Either classification algorithm requires a training phase before it can be used in the process shown in Figure 3. However, instead of passing along the commands to the robot in order the replicate the human movement. The human intention of moving a left / right arm in combination with the current context of the worker's position and task can be evaluated (5). Then it can be integrated into the safety-system. While vision-based safetysystems must detect differences in arm positions and therefore reacting relatively late, the main advantage of the EEGintention recognition is that the processing of brainwave signals can already begin before the actual movement is executed. After the evaluation-phase (5), there is the opportunity to follow up with a reaction (6). Based on Villani et al. (2018) there are three possible feedback options. The first option is to fully stop the robot. The second alternative is to reduce speed. A third possibility is to reduce power and force in order to not injure human workers [9]. While the first three steps within the process stay the same (measuring, filtering and feature extraction), there are opportunities to train and later to use different classification algorithms. Bousseta et al. (2017) measured the arm-movement intentions for four participants. The overall mean accuracy achieved is $84.18 \%$. In their paper similar results presented had an accuracy of $78 \%$ up to $88 \%$. However, in each case the method for performing a classification was based on SVM's [5].

Due to the rapid development of machine learning algorithms including artificial neural networks and deep learning, there are more advanced classifiers available than SVM's.

Jiao et al. (2017) compared different classifiers for EEGmeasured data. Their accuracy for the SVM with $84.66 \%$ is close to the aforementioned $84.18 \%$. In their paper, a model based on a Convolutional Neural Network increased the accuracy up to $92.37 \%$ [14]. However, according to Géron (2017), there are two main approaches of deep learning: Recurrent Neural Networks (RNN) and Convolutional Neural Networks (CNN). CNN's are typically used for image recognition and visual feature based identification, RNN's, on the other hand, are typically used to identify time-based data series such as stock-market rates and language recognition [15]. Due to EEG-signals for arm-movements also being timebased series and since they can, similarly to language recognition, be seen as a means of communication there is also the opportunity of increasing accuracy and recall by using RNN's [15]. In the context of safety in a human-robot collaboration scenario, it is important to implement bias towards high recall over high accuracy when training the classifiers to recognise all movement intentions. On the other hand, a false-alarm-scenario is a False-Positive intention recognition leading to an unnecessary interruption of the robot movement. This is also desired to be prevented or kept as low as possible, since it will slow down the assembly process.

\section{CONCLUSION}

In shared manufacturing environments, human-robot communication becomes more important. While robot movements are predefined, human intentions are difficult to detect. Video-based safety systems can only react to human movement after it started. A novel concept of measuring human movement intentions and integrating them into a safety system is presented. It is expected to provide additional reaction time to safety systems. The proposal is to use existing approaches that demonstrated the use EEG-measured brainwaves to replicate human movements with robotic arms. By using a similar approach to use the brainwaves as a means of communication by detecting human intention to move. The main goal is to validate the possibility to detect human intentions and by providing this information to the robot enable the vision of symbiotic human-robot collaborations.

\section{REFERENCES}

[1] R. Palmarini et al., "Designing an AR interface to improve trust in Human-Robots collaboration," Procedia CIRP, vol. 70, pp. 350355, 2018.

[2] I. F. of R. (IFR), "IFR. Executive summary world robotics 2016 industrial robots. Technical Report.," 2016.

[3] P. Ferreira, S. Doltsinis, and N. Lohse, "Symbiotic assembly systems - A new paradigm," Procedia CIRP, vol. 17, pp. 26-31, 2014.

[4] R. J. Halme, M. Lanz, J. Kämäräinen, R. Pieters, J. Latokartano, and A. Hietanen, "Review of vision-based safety systems for human-robot collaboration," Procedia CIRP, vol. 72, pp. 111-116, 2018.

[5] R. Bousseta, I. El Ouakouak, M. Gharbi, and F. Regragui, "EEG Based Brain Computer Interface for Controlling a Robot Arm Movement Through Thought," Irbm, vol. 39, no. 2, pp. 129-135, 2018.

[6] P. Gustavsson, M. Holm, A. Syberfeldt, and L. Wang, "Humanrobot collaboration - Towards new metrics for selection of communication technologies," Procedia CIRP, vol. 72, pp. 123$128,2018$.

[7] G. Weichhart, M. Åkerman, S. C. Akkaladevi, M. Plasch, Å. FastBerglund, and A. Pichler, "Models for Interoperable Human Robot Collaboration," IFAC-PapersOnLine, vol. 51, no. 11, pp. 36-41, 2018.

[8] International Organization for Standardization, "ISO 102181:2011," Safety requirements for industrial robots, 2011. [Online] Available: https://www.iso.org/standard/51330.html. [Accessed: 13-Dec-2018].

[9] V. Villani, F. Pini, F. Leali, and C. Secchi, "Survey on humanrobot collaboration in industrial settings: Safety, intuitive interfaces and applications," Mechatronics, no. June 2017, pp. 119, 2018.

[10] P. Gustavsson, A. Syberfeldt, R. Brewster, and L. Wang, "Humanrobot Collaboration Demonstrator Combining Speech Recognition and Haptic Control," Procedia CIRP, vol. 63, pp. 396-401, 2017.

[11] A. Mohammed and L. Wang, "Brainwaves driven human-robot collaborative assembly," CIRP Ann., vol. 67, no. 1, pp. 13-16, 2018 .

[12] H. Liu and L. Wang, "Human motion prediction for human-robot collaboration," J. Manuf. Syst., vol. 44, pp. 287-294, 2017.

[13] D. Planelles, E. Hortal, Á. Costa, A. Úbeda, E. Iáñez, and J. M. Azorín, "Evaluating classifiers to detect arm movement intention from EEG signals," Sensors (Switzerland), vol. 14, no. 10, pp. 18172-18186, 2014

[14] Z. Jiao, X. Gao, Y. Wang, J. Li, and H. Xu, "Deep Convolutional Neural Networks for mental load classification based on EEG data," Pattern Recognit., vol. 76, pp. 582-595, 2018.

[15] A. Géron, Hands-On Machine Learning with Scikit-Learn and TensorFlow. O'Reilly Media, 2017. 